\title{
FAULTS DETECTION BASED ON FUZZY CONCEPTS FOR VIBRATIONS MONITORING IN GAS TURBINE
}

\author{
Bachir ALILI ${ }^{1,2,4}$, Ahmed HAFAIFA ${ }^{1 *}$ Abdelhamid IRATNI $^{3}$ \\ ${ }^{(1)}$ Gas Turbine Joint Research Team, University of Djelfa, Algeria \\ (2) Applied Automation and Industrial Diagnostics Laboratory, University of Djelfa, Algeria \\ ${ }^{(3)}$ Faculty of Science and Technology, University of Bordj Bou Arreridj, 34030 DZ, Algeria \\ ${ }^{(4)}$ University Lorraine, France \\ E-mails: $\underline{\text { b.allili@ univ-djelfa.dz, a.hafaifa@univ-djelfa.dz, iratni@univ-bba.dz }}$
}

\begin{abstract}
The use of new technologies in modern industry improves productivity but induces complexity in the industrial system. This complexity makes it vulnerable to faults, which requires significant expense in terms of safety, reliability and availability. Indeed, a diagnostic operation is essential for the operational safety and availability of these industrial systems. This diagnostic operation is based on two important functions which are the detection and localization of anomalies, which consists to verifying the consistency of the data taken in real time from the installation with a reliable model, to ensure the good performance of the monitoring system. Hence, the diagnosis of gas turbines is a main component for making maintenance decisions for this type of machine. In this paper, the faults detection approach based on fuzzy logic is applied for the vibrations monitoring of a gas turbine, in order to monitor their operating state by including the detection and occurrence of vibration faults, thus using determined fault indicators based on the input / output variables of the examined gas turbine. In this work, the investigation results of fuzzy fault detection approach applied on gas turbine vibration are presented, based on the actual data recorded in the different gas turbine operating modes. However, analysis of the defect detection results was performed in order to determine the influence of these vibration defects on the deferent operating modes of the examined machine. This makes it possible to find the causes of failures and then to deduce the actions to follow the operational safety of the examined turbine.
\end{abstract}

Keywords: Faults Detection, Fuzzy Logic, Decision Making, Vibration Monitoring, Gas Turbines.

\section{INTRODUCTION}

Increasing the productivity, quality and availability of industrial systems are major issues, essentially linked to the safety and operational safety of these industrial facilities (safety of people, the environment and equipment). Indeed, several modern approaches have been designed to control the operational safety of industrial systems, and which also aim to increase production rates while reducing maintenance time. These methods focus on carrying out an effective diagnosis of these systems hampered by faults or malfunctions.

The objective of this work is to understand the growing complexity of gas turbine systems and to realize a fault detection approach based on fuzzy concepts for the monitoring of vibrations in this type of rotating machine. This, allows the reduction of the maintenance cost in these gas turbine installations, by a adequate monitoring approach, using to the proposed fault detection indicators for improving the efficiency of gas turbines. Therefore, economic impact given by the diagnosis of this system in terms of availability and production.

Adel Alblawi in [1] proposed an approach to diagnosing faults in an industrial gas turbine based on the thermodynamic model coupled to an artificial neural network. Ahmed Zohair Djeddi et al. in [2] explored reliability algorithms using the modified Weibull distribution applied to a gas turbine for maintenance purposes. Also, Cristiano Hora Fontes and Hector Budman in [5] carried out a hybrid clustering approach for multivariate time series for the analysis of failures in a gas turbine, and Ehsan Mohammadi et al. in [7] identified the failures of a gas turbine using a fuzzy logic-based detection system for a deterioration in performance.

Mohamed Benrahmoune et al. in [18] proposed a method for detecting and modeling the vibrational behavior of a gas turbine with an approach of dynamic neural networks and Marcin Adamowicz and Grzegorz Zywica in [17] proposed an advanced health monitoring system of gas turbines. 
Indeed, the detection of faults based on fuzzy logic represents a scientific and industrial challenge due to the industrial equipment complexity, founded by the economic interest of the effectiveness of these fuzzy diagnostic strategies. Several works have dealt with the theme of industrial supervision based on fuzzy concept for the management of failures in different industrial applications, thus accurately posing the interest of these diagnostic strategies resulting from artificial intelligences; Chuanlai Yuan et al in [4] have proposed a fault diagnosis method for power distribution networks applications based on Petri nets and fuzzy logic and Toufik Berredjem et al in [26] have performed a faults diagnosis strategy applied to rolling using an expert fuzzy system. Also, Imad Eddine Kaid et al in [13] carried out a failure diagnostic strategy of a photovoltaic system based on an adaptive neuro-fuzzy inference approach applied to a solar power plant.

In this work, a failure detection approach based on fuzzy logic is applied for monitoring the vibrations of a gas turbine as well as their operating state by including the detection and the appearance of vibration faults using fault indicators developed using turbine input / output variables. In this context, we propose the use of fuzzy TakagiSugeno models for the development of a fault detection strategy for the studied system. This strategy is based on a comparison of the estimated error between the state of the faulty system and the state of the reference system in order to estimate the performance of the fuzzy diagnostic strategy.

Experimental results will be illustrated for each type of fault using tests performed in real time, on the gas turbine system at Hassi R'mel station in south of Algeria, using the proposed fuzzy diagnostic strategy. We will first analyze the structure of the fuzzy approach; Then, we will give the results of the tests carried out on the examined gas turbine. Finally, we will draw the necessary conclusions regarding the use of this fuzzy diagnostic strategy on the tested industrial turbine.

\section{FUZZY DIAGNOSTICS}

The diagnosis of gas turbines is generally carried out through knowledge of its normal behavior, total control of the different operating modes is then essential when considering an advanced diagnosis of this type of machine [9, 11, 14, 16, 20, 27, 29]. Indeed, the application of fuzzy logic techniques improves the reliability of the monitoring system and the sensitivity of fault detection, it is also capable of causing, in severe failures, a turbine stop or allowing the system to continue to operate in degraded mode in the event of a problem which does not require an immediate stop $[2,6,8,10,12$, 22, 24, 28, 30].

In this section, the steps for carrying out a fuzzy diagnostic approach will be presented in the order of formalizing a mechanism for exploiting them for monitoring a gas turbine. For this, knowledge a priori on the turbine is represented by a set of rules and facts, which constitute what is called the knowledge base, as it is shown in Figure 1. This base is constructed using formalization assistance tools strongly linked to turbine operation.

To apply this fuzzy concept to turbine monitoring, the operating data of this machine will then be fuzzified and analyzed by the rule base of the fuzzy system. The ground rules will give us an answer as the input data, which will be defuzzified to generate the turbine diagnostic signals. To characterize the operating mode of the turbine in real time, a fuzzy Takagi-Sugeno type modeling is proposed, recording information, recognizing and indicating anomalies in the behavior of this rotating machine.

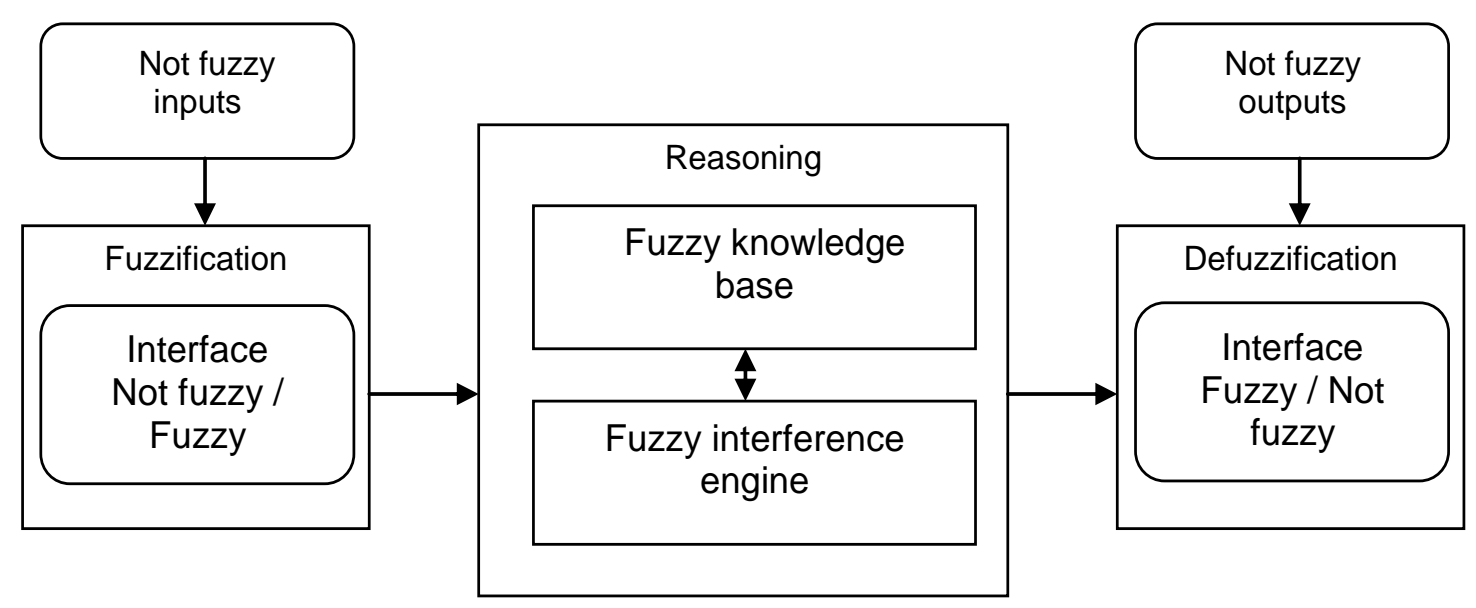

Fig. 1. Fuzzy concept structure 
Fuzzy Takagi-Sugeno (TS) modeling is a mathematical representation of non-linear systems [61]. By restricting itself to a compact set of state variables, a fuzzy TS model can represent exactly a nonlinear system by a collection of linear submodels weighted through nonlinear functions called "Membership Functions". The activation of each sub-model is governed by "If _ Then" rules in the following form $R_{i}$ [21, 23]:

Rulei:

$$
\begin{gathered}
\text { If }\left(\mathrm{z}_{1}(\mathrm{t}) \text { is } \mu_{\mathrm{i} 1}\right) \text { and }\left(\mathrm{z}_{2}(\mathrm{t}) \text { is } \mu_{\mathrm{i} 2}\right) \text { and.... }\left(\mathrm{z}_{\mathrm{p}}(\mathrm{t}) \text { is } \mu_{\mathrm{i} 1 \mathrm{p}}\right) \\
\text { Then } \\
\left\{\begin{array}{l}
\dot{x}(t)=A_{i}(x(t)) x(t)+B_{i}(x(t)) u(t) \\
y(t)=C_{i}(x(t)) x(t)+D_{i}(x(t)) u(t)
\end{array}\right.
\end{gathered}
$$

where $R_{i}$ are the fuzzy rules, $i \in\{1,2, \ldots, r\}$ is the number of rules in the model, $\mu_{i j j}$ are the membership functions with $j \in\{1,2, \ldots, p\}, \quad z_{p}$ represent the premise variables, $A_{i}(x(t)) \in R^{n_{x} \times n_{x}}$, $B_{i}(x(t)) \in R^{n_{x} \times n_{u}}, C_{i}(x(t)) \in R^{n_{y} \times n_{x}} \quad$ and $D_{i}(x(t)) \in R^{n_{y} \times n_{u}}$ are the system state matrices.

In order to interpret each rule $R_{i}$, the operator (and) is defined as the multiplication operator, from this consideration, each ith sub-model has its degree of belonging to the global model given by:

$$
w_{i}(z(t))=\prod_{i=1}^{p} u\left(\mu_{i j}\right)
$$

with $u\left(\mu_{i j}\right) \in[0,1]$ is the degree of belonging of the premise $z_{i}(t)$.

The global TS model is obtained using the weighted sum given by $[3,15]$ :

$$
\left\{\begin{array}{l}
\dot{x}(t)=\frac{\sum_{i=1}^{r} w_{i}(z(t))\left(A_{i}(x(t)) x(t)+B_{i}(x(t)) u(t)\right)}{\sum_{i=1}^{r} w_{i}(z(t))} \\
y(t)=\frac{\left.\sum_{i=1}^{r} w_{i}(z(t))\left(C_{i} x(t)\right) x(t)+D_{i}(x(t)) u(t)\right)}{\sum_{i=1}^{r} w(z(t))}
\end{array}\right.
$$

We get:

$$
h(z(t))=\frac{w_{i}(z(t))}{\sum_{i=1}^{r} w_{i}(z(t))}
$$

The standard TS model for the system is given in the following form:

$$
\left\{\begin{array}{l}
\left.\dot{x}(t)=\sum_{i=1}^{r} h_{i}(z(t))\left(A_{i} x(t)\right) x(t)+B_{i}(x(t)) u(t)\right) \\
y(t)=\sum_{i=1}^{r} h_{i}(z(t))\left(C_{i}\left(x(t)+D_{i}(x(t)) u(t)\right)\right.
\end{array}\right.
$$

Hence $h_{i}(z(t))$ represent the activation function of the ith rule of the fuzzy model, for $i=1,2, \ldots, r$, these functions verify the property of a convex sum given by:

$$
\left\{\begin{array}{l}
h_{i}(z(t)) \geq 0 \forall_{i} \\
\sum_{i=1}^{r} h_{i}(z(t))=1
\end{array}\right.
$$

Finally, the defuzzification of the fuzzy model makes it possible to obtain the state representation of a nonlinear model by the interconnection of local models' invariant in time by nonlinear activation functions [21, 25]. To estimate the parameters of the turbine examined, an identification from input / output data is proposed, based on the minimization of a cost function calculated from the difference between the estimated output of the fuzzy system TS and the measured output $y(t)$ of the system.

\section{GAS TURBINE FAILURES DETECTION}

The fuzzy logic makes it possible to take into account the inaccuracies inherent in the data and to account for the expression of the rules which make it possible to formulate a diagnosis to determine the monitoring actions for the turbine. In this paper, the measurements were carried out on the bearings of a gas turbine of the TORNADO type Siemens SGT200, installed in the south of Algeria, from mobile accelerometers and the sensors mounted on this turbine.

Forecasting is the prediction of performance parameters and measured turbine values using a diagnostic system capable of determining the moments when an overshoot or decrease in nominal operating values. This can be used to optimize the maintenance planning of the turbine. However, the determination of the maintenance functions based on the planning of the actions makes it possible to maintain and restore the state of good functioning of the gas turbine and to ensure their availability for production with preservation of the environment and safety by optimal costs.

In this work, the vibration detection inductors will be determined by the real-time diagnostic function (turbine inspections in operation. This is to have maximum productivity of gas turbine reviews, these fault inductors allow rapid control of different turbine system zones; Turbine section, axial compressor section and combustion section. 


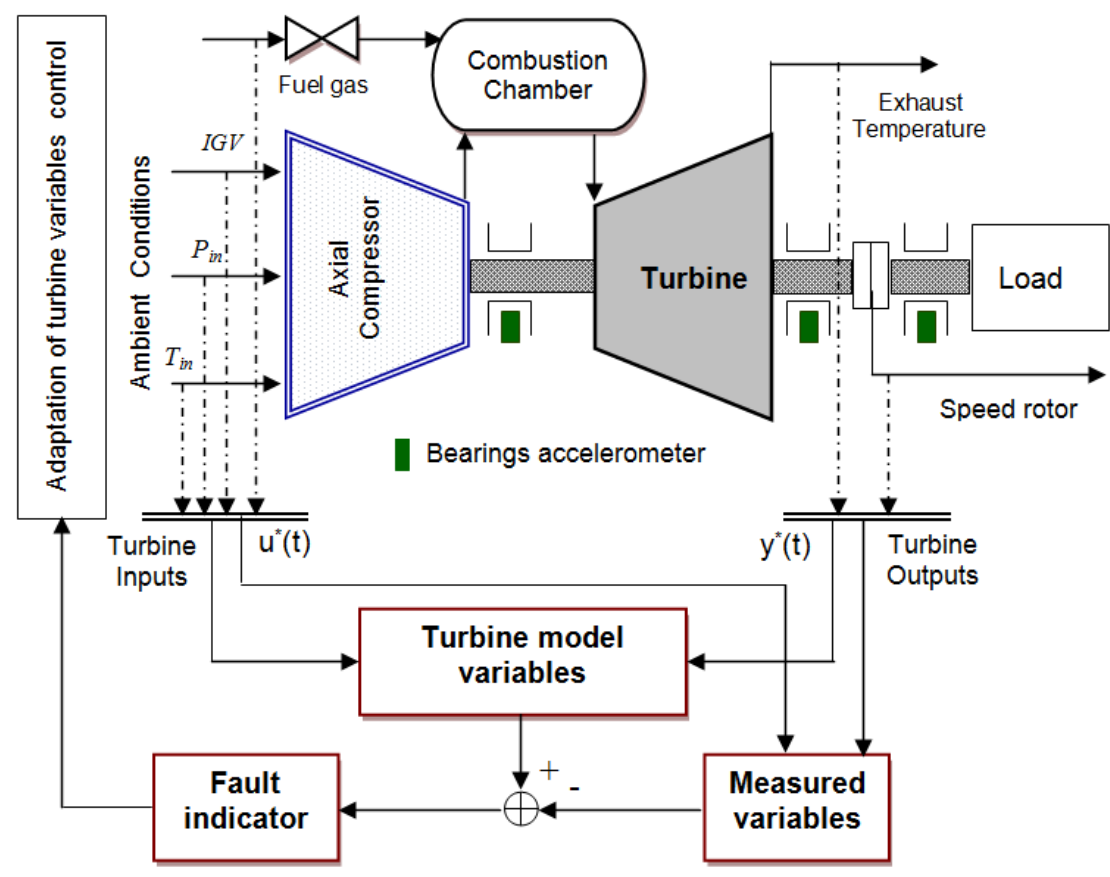

Fig. 2. Gas turbine failure detection strategy

These consist of continuous and general monitoring of the unit and its auxiliaries with the turbine running. It is advisable to record the main parameters during the first start-ups and when the turbine is running. Indeed, this operation is used to have reference values on the performance parameters, when the machine is new. This will allow a better assessment of any change in the operation of the gas turbine during its lifetime and will help to discover the causes of the possible faults and to choose the appropriate solution.

The operating data must be acquired in the transient phases (starting, stopping) and under steady state conditions. The main parameters of the on-the-go inspection are; rotation speed, load, number of starts, number of operating hours, pressure and temperature at different turbine operating points, ambient temperature and pressure, oil and fuel pressure as well as the different filter and vibration signals.

The purpose of the detection procedure is to determine the appearance and the instant of occurrence of a fault. To reach this objective, one uses residues which are obtained by comparing the behavior of the model of the system to that of the real system. The residuals are representative of the differences between the observed behavior of the system and the reference behavior expected when the system operates normally. These residues are generally at zero average and have a variance determined in the absence of malfunctions. A generic way to construct a residue is to estimate the output vector $y($.$) of the system.$
The estimate $\hat{y}($.$) is then subtracted from the$ output signal $y($.$) in order to form the following$ residue vector $[23,26]$ :

$$
r(k)=y(k)-\hat{y}(k)
$$

In the presence of faults, the signal $r($.$) , thus$ formed will deviate significantly from the value zero and will be identical to zero when the system is operating normally.

In practice, the residue does not have exactly a zero value in the absence of defects because, during the modeling phase, several simplifying hypotheses are introduced leading to a model which does not faithfully reflect the real system $[6,8]$. In addition, the measurements made on the system are most often marred by measurement noises. The residue vector is then written:

$$
r(k)=y_{m}(k)-\hat{y}(k)
$$

Where $y_{m}(k)$ is the measured output of the system which is composed, in addition to the actual output, of noises of various natures relating to the instrumentation and the modeling uncertainties. In this situation, an elementary detection method consists in comparing the value of the residue with a predefined threshold $\varepsilon$ (function of modeling errors). An alarm is triggered each time this threshold is crossed [17-18]:

$$
\left\{\begin{array}{l}
r(k) \leq \varepsilon \Leftrightarrow d(k)=0 \\
r(k) \prec \varepsilon \Leftrightarrow d(k) \neq 0
\end{array}\right.
$$

Where $d($.$) represents the vector of the defects.$

We can also model the residue as a random variable distributed according to a normal law. 
Statistical tests are thus used at this level making it possible to detect changes in the statistical characteristics of the residue.

\subsection{Vibration measurement system}

The vibration measurements allow a diagnosis of sudden vibrations by the machines, in order to identify their malfunctions and to repair them. In fact, in most vibration measurement applications, parameters, acceleration, speed and displacement are information that it is essential to know with the best possible precision, particularly for the design of diagnostic and monitoring strategies for the rotating machine.

The role of the sensors is to transform the vibrations at the measuring points into an electrical voltage. Hence, the sensitivity of the sensor gives the ratio between the measured voltage and the proper vibration, for the examined gas turbine system in this work, three types of vibration sensors are used, for predictive maintenance are the displacement sensors, inductive speed sensors, and accelerometers.

\section{Displacement sensor}

The proximity (displacement) sensor, shown in Figure 3, measures the relative shaft vibrations of the rotating machine. It is also used to measure other parameters such as axial position and phase reference. It provides a voltage proportional to the distance between the sensor head and the target. The main criteria for selecting a proximity sensor are:

- Sensitivity,

- The measurement distance.

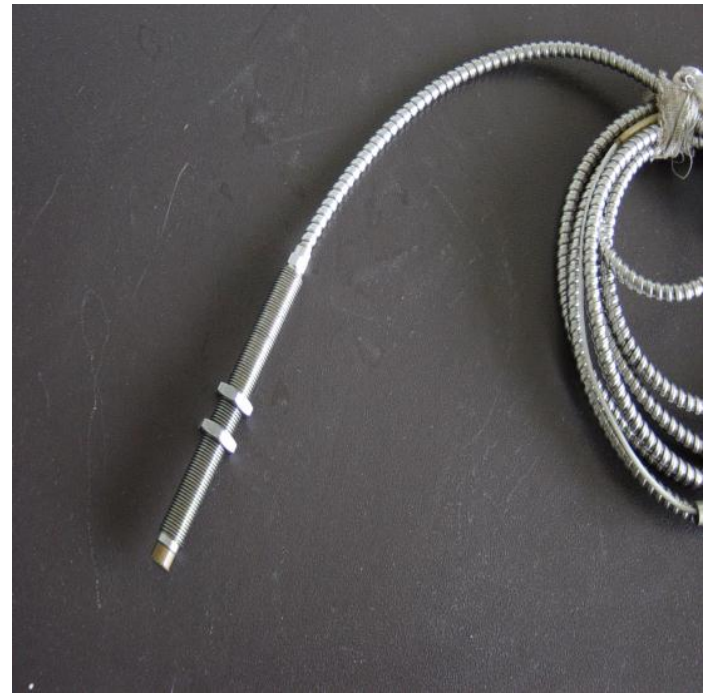

Fig. 3. Proximity (displacement) sensor

\section{Speed sensor (Velocimeter)}

The speed sensor is a velocimeter type, as it is shown in the Figure 4, this sensor makes it possible to measure the vibration speeds of the bearings of a rotating machine. The velocimeter has the advantage of providing a direct velocity of the vibration velocity without the use of a conditioner or amplifier.

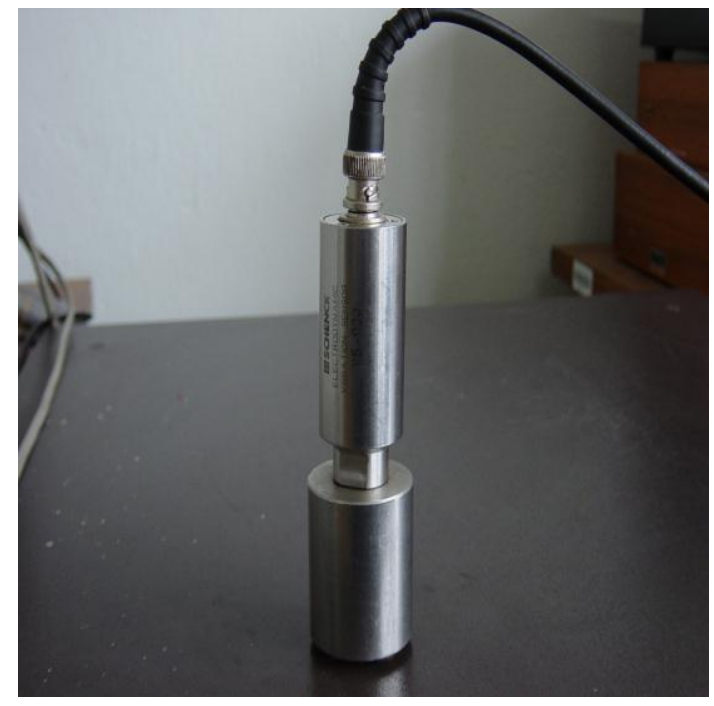

Fig. 4. Speed sensor (Velocimeter)

\section{Acceleration sensor (accelerometer)}

The acceleration sensor, shown in Figure 5, is very often best suited for measuring vibration and shock. Indeed, an acceleration measurement can always make it possible to know the speed and the displacement by simple or double integration, the reverse path not being desirable, given the degradation of the signal / noise ratio inherent in the bypass operation. These acceleration sensors are motion sensors that do not need a reference point.

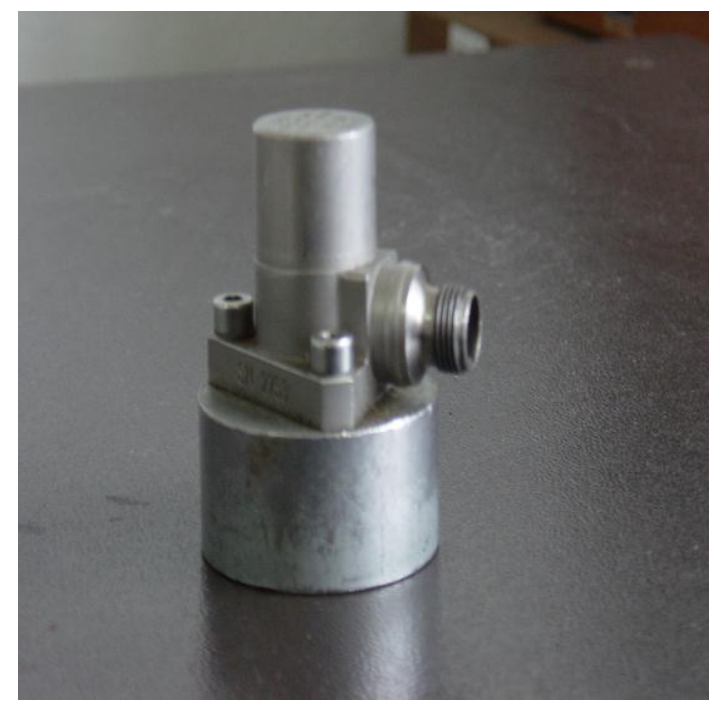

Fig. 5. Acceleration sensor (accelerometer)

Other sensors such as the phase sensor that can be used, these sensors find their application in balancing, resonance research and monitoring of rotating machines. The phase reference sensor, as shown in Figure 6, is a photocell or non-contacting sensor that detects one pulse per revolution. 


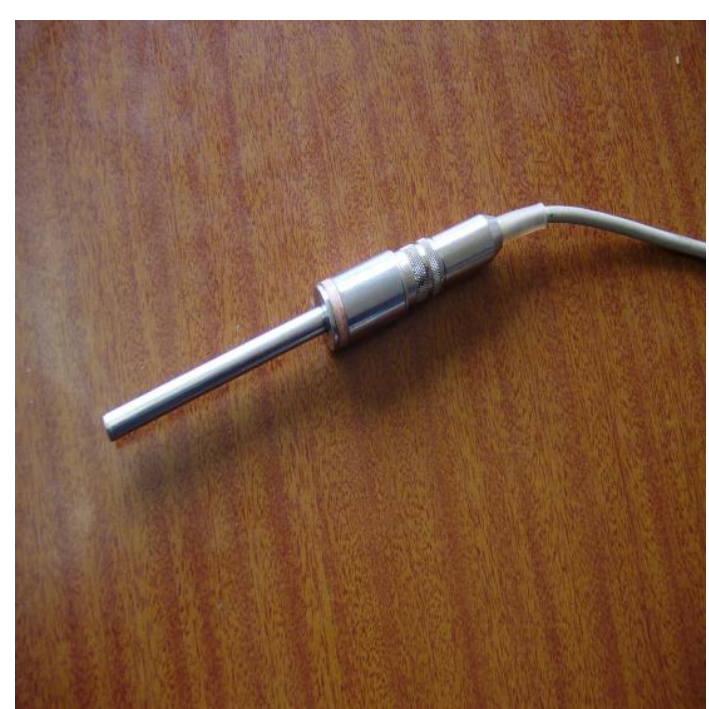

Fig. 6. Phase sensor

\section{INVESTIGATIONS RESULTS}

This section is devoted to the development of fault detection tools applied to a gas turbine, which will be used for their maintenance. We develop the fault diagnosis approach caused by the vibrations of this machine based on fuzzy logic, in order to validate this method, to ensure a functional availability of the examined system and to ensure a reliable diagnostic function to avoid unforeseen failures in this gas turbine system.

The role of supervision is to monitor the effect of variations in turbine parameters in operation, it is then a question of monitoring that the turbine is in normal operation. If it is not, it acts at least to avoid damage to the installation, to limit untimely stops and at best to guarantee the continuity of operation of this rotating machine. In this section, four turbine operating situations are presented; the first situation is in normal operation, the second situation is in abnormal operation in the case of $1^{\text {st }}$ alarm, the third situation in abnormal operation in the case of $2^{\text {nd }}$ alarm with operation in degraded mode and the last situation in abnormal operation in the case of $3^{\text {rd }}$ alarm before total turbine shutdown. In these different situations, the vibrations variation and the vibrations signal response and diagnostic function associated with each situation are presented.

\subsection{Fuzzy variables input / output of the examined gas turbine}

The gas turbines availability in gas transportation facilities is crucial to ensure optimal and reliable production and transportation. Hence, premature failure can lead to substantial economic losses, due to unplanned outages and possible damage to these facilities. However, the operating experience of operators of this type of machine leads to the collection of acquired knowledge to the monitoring of this equipment.

In this section, the variables thresholds was determined using the feedback knowledge of the operators of the turbine under consideration. By adding the turbine manufacturer's recommendations with the use of standards (ISO 7919-2-2001, ISO 10816-4, ISO 10816-6), in order to propose monitoring solutions adapted to the examined turbine diagnostic strategy.

From the vibration levels, the functions of the fuzzy system can be structured, with the fuzzification of turbine variables. The fuzzy linguistic variables are defined as follows:

For the vibration levels

- Low, vary in the interval $[0.65,6.11] \mu \mathrm{m}$

- Medium, vary in the interval [6, 11-35.4] $\mu \mathrm{m}$

- High, vary in the interval of the values greater than $[15.4,150] \mu \mathrm{m}$

For temperature variation

- Low, vary in the range $[25,68]^{\circ} \mathrm{C}$

- Averages, vary in the range $[68,75]^{\circ} \mathrm{C}$

- Raised, vary above the value $75^{\circ} \mathrm{C}$

For the variation of the maintenance history

- Low, vary in the interval $[0,3]$

- Average, vary in the interval [3, 7]

- High, vary in the interval of the values greater than [7]

The input / output variables of the gas turbine examined are presented as follows:

- UD10X: Vibrations Input X of gas generator,

- UD10Y: Vibrations Input Y of gas generator,

- UD11X: Vibrations Output X of gas generator,

- UD11Y: Vibrations Output Y of gas generator,

- UD12X: Vibrations Input X of power turbine,

- UD12Y: Vibrations Input Y of power turbine,

- UD13X: Vibrations Output X of power turbine,

- UD13Y: Vibrations Output Y of power turbine,

- Average temperature,

- History of maintenance.

The diagnostic variable output has the following values associated with the speech universe:

- Danger, vary in the range $[0,30]$,

- Maintenance, vary in the range [30, 50],

- Warning Alarm, vary in the range [50, 70],

- Normal, vary in the range [70, 100].

\subsection{Operating situations of the examined turbine}

The gas turbine monitoring systems based on the measured values of these variables allows the detection functions of this machine to be generated. Figure 7 shows the vibration signals of the turbine in normal operation. These data and according to the maintenance history of this equipment and the relatively low number of interventions, ten interventions over a period of two years, allowing the maintenance history to be low as shown on the variation of the diagnostic function output given in Figure 8. Hence Figure 9 shows the variation of turbine vibration output in the normal operation mode.

In Figures 7, 10, 13 and 16, the causes of the sudden increases in the level of the measured 
signals is explained by that the first level is a starting peak is due to the starting sequences of the machine and the second level occurs due to the opening of the recycling valve (reduction in the quantity of gas in the machine), which may be occur again if this recycling valve is closed.

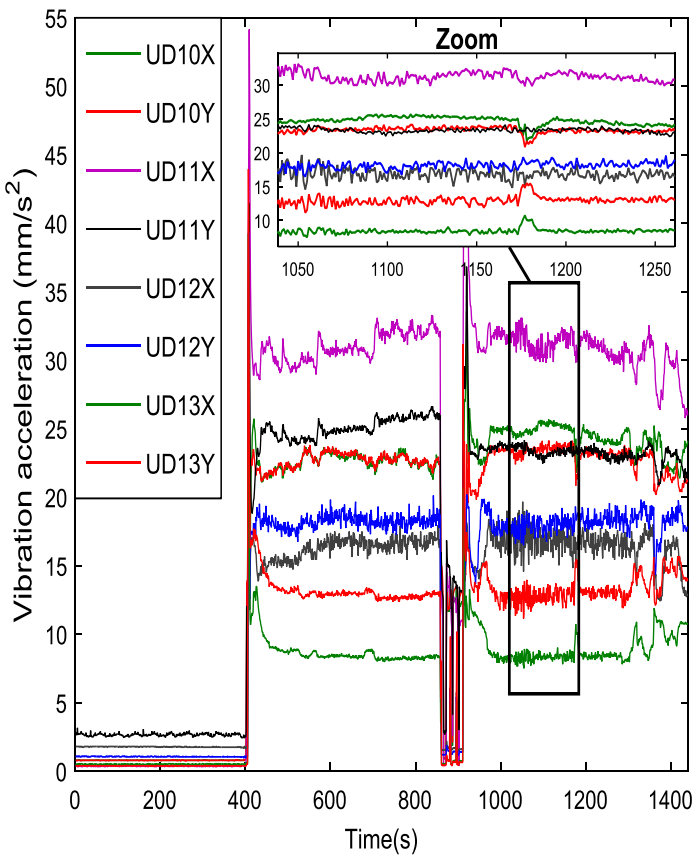

Fig. 7. Turbine vibration signals normal operation

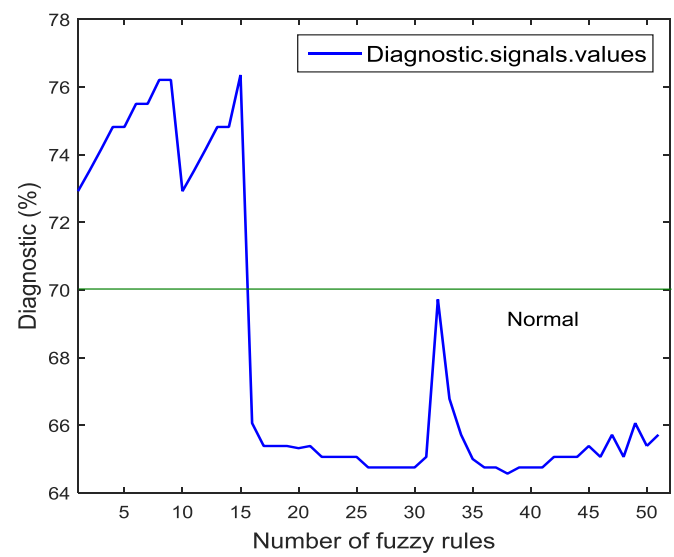

Fig. 8. Variation of diagnostic function output in normal operation

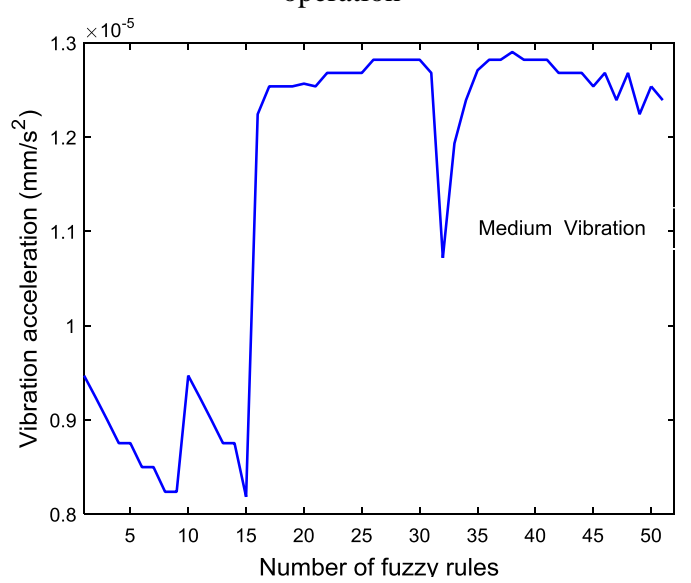

Fig. 9. Variation of vibration output in normal operation
Figure 10 shows the vibration variations of the turbine abnormal operation in the case of the $1^{\text {st }}$ alarm, the vibration levels are low but have $1^{\text {st }}$ order faults. Hence Figure 11 shows the variation of turbine vibration signals in the abnormal operating mode in this case of $1^{\text {st }}$ alarm and Figure 12 shows the variation of diagnostic function output for this operating mode.

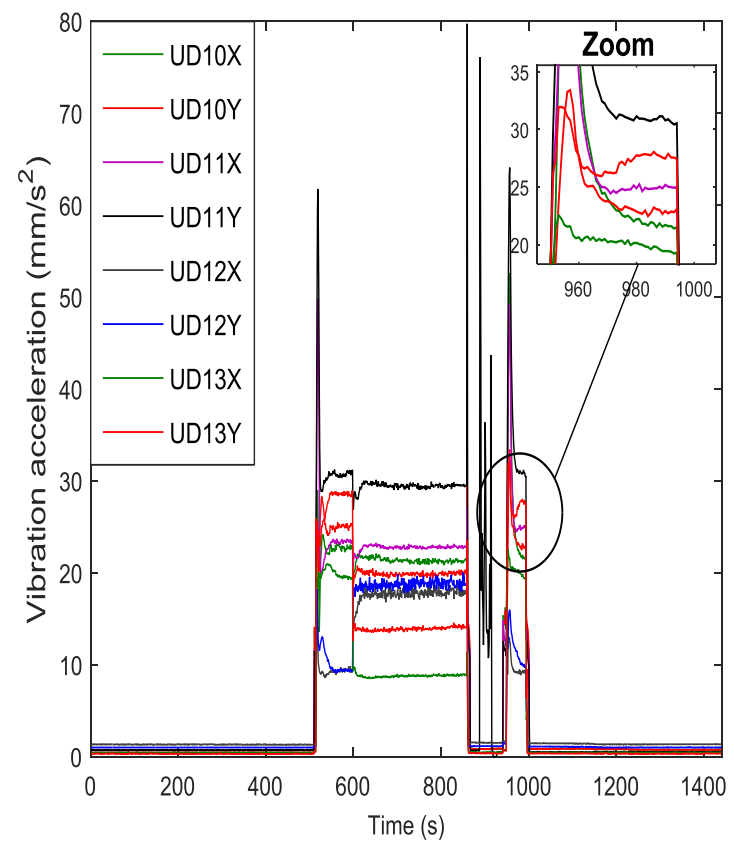

Fig. 10. Turbine vibration signals abnormal operation $1^{\text {st }}$ alarm

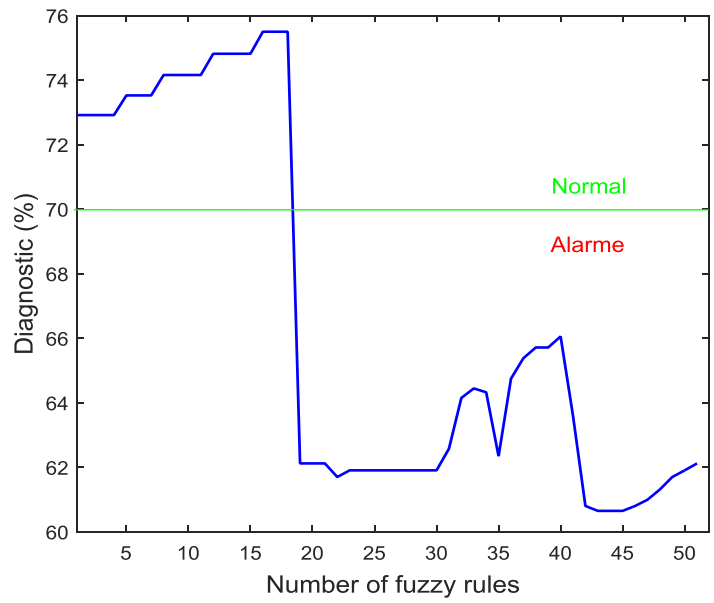

Fig. 11. Turbine vibration signals abnormal operation in case of $1^{\text {st }}$ alarm

With the increase in the speed of rotation of the turbine, we see in Figure 13 the variation of vibration signals from the turbine abnormal operation $2^{\text {nd }}$ alarm in degraded mode operation. It has been observed that with the increase in the vibration levels shown in Figure 14, the diagnostic function indicates the status of the $2^{\text {nd }}$ order alarm, as shown in Figure 15. 


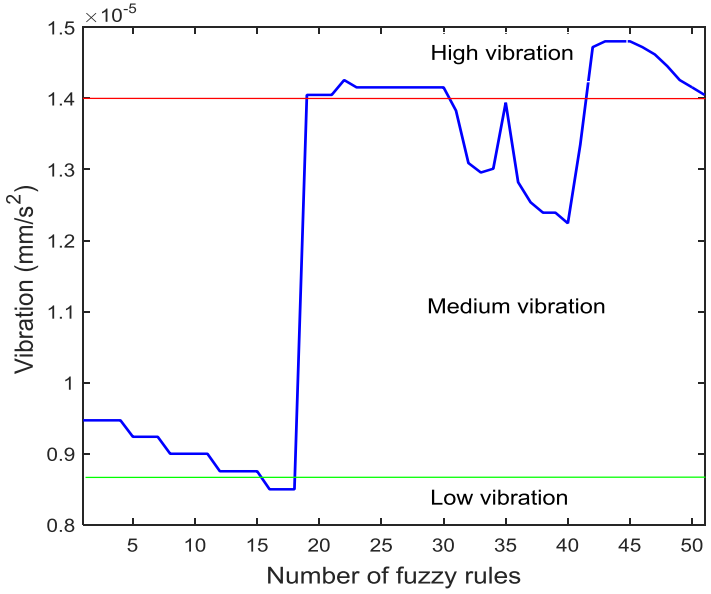

Fig. 12. Abnormal vibration output variation in case of $1^{\text {st }}$ alarm

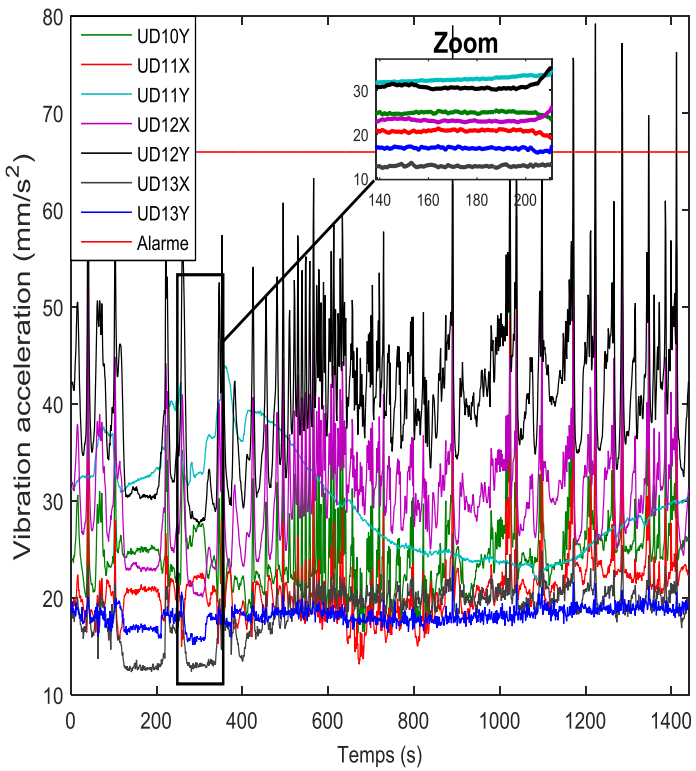

Fig. 13. Turbine vibration signals abnormal operation $2^{\text {nd }}$ alarm, degraded mode operation

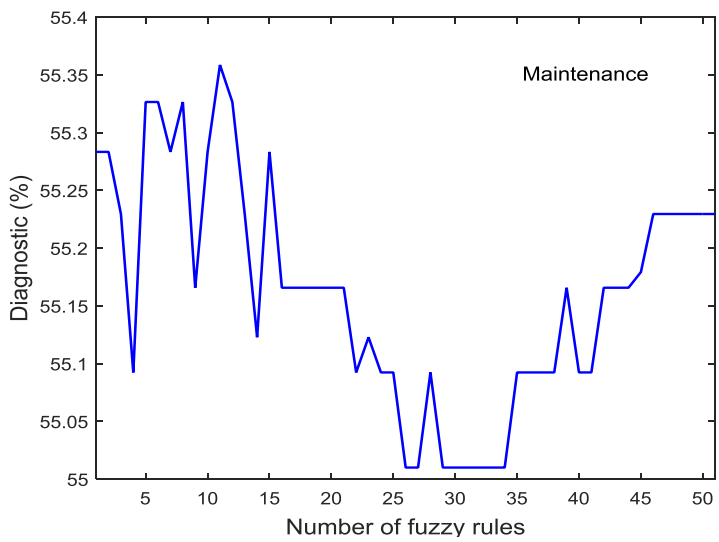

Fig. 14. Turbine vibration signals abnormal operation in case of $2^{\text {nd }}$ alarm, operation in degraded mode

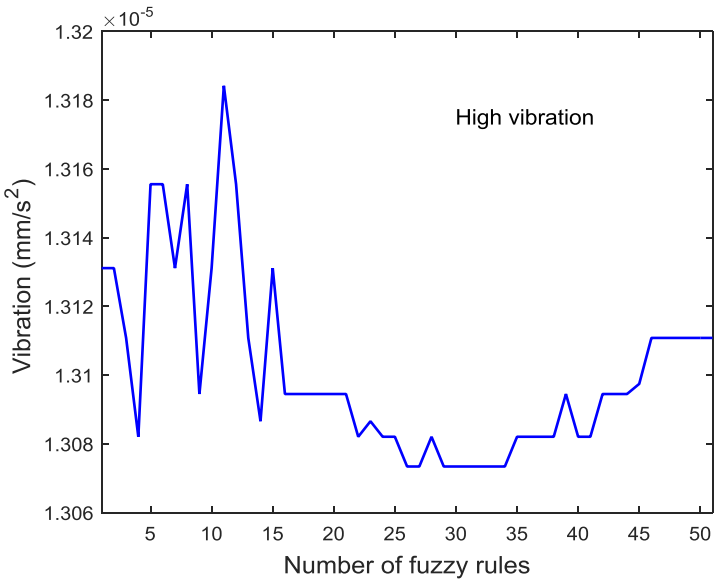

Fig. 15. Abnormal vibration output variation in case of $2^{\text {nd }}$ alarm, operation in degraded mode

The last case presents a 3rd order danger situation which forces the turbine to stop operating as shown in Figure 16. Indeed, the vibration variation is very high, as shown in Figure 17, implies that the diagnostic function indicates this danger situation, as shown in Figure 18.

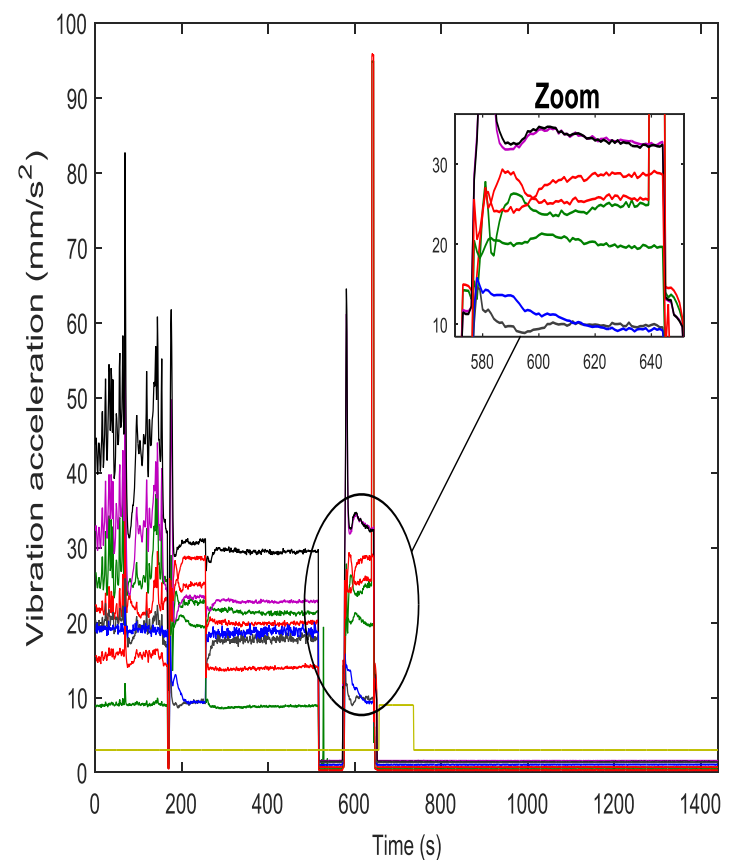

Fig. 16. Turbine vibration signals abnormal operation in case of 3rd alarm before turbine shutdown

The analysis of the vibration data in the gas turbine examined is used to collect the data and to make the various treatments and its scaling. For our application, the measurement points were made on the gas turbine system, from mobile accelerometers on the turbine bearings. The results obtained are satisfactory, hence the rules of the fuzzy structure succeed in expressing the knowledge of the experts of maintenance of the turbine as regards the detection of vibration level. 


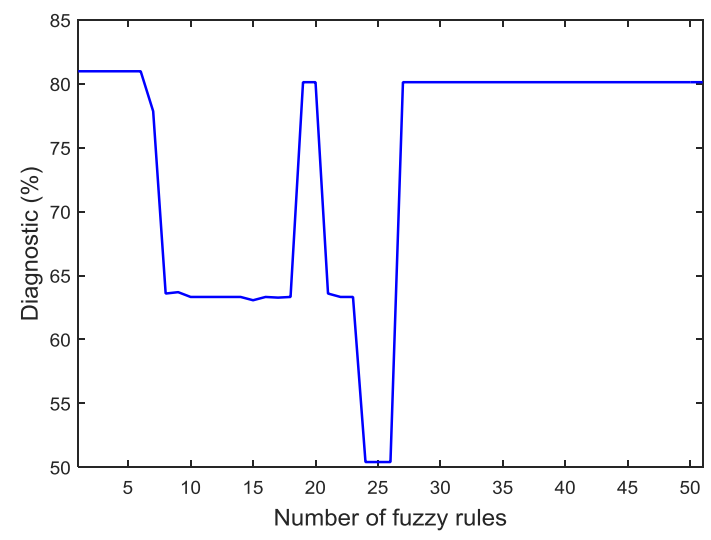

Fig. 17. Turbine vibration signals abnormal operation in case of 3rd alarm before turbine shutdown

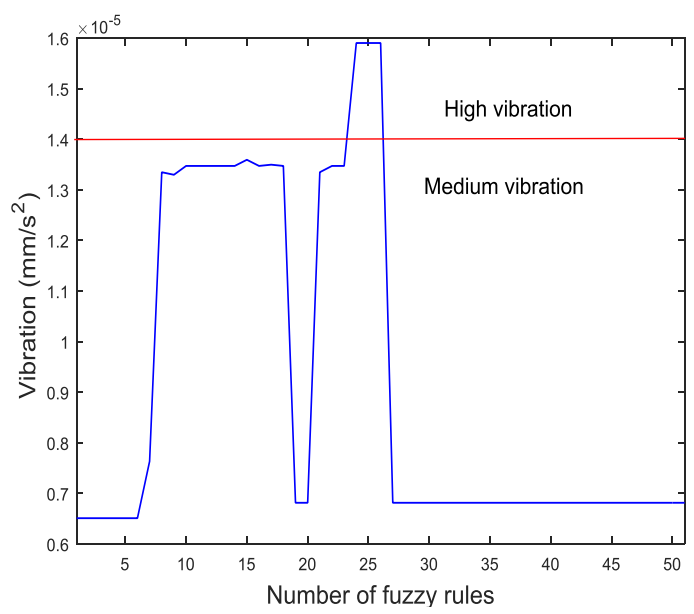

Fig. 18. Abnormal vibration output variation in case of $3^{\text {rd }}$ alarm before turbine shutdown

\section{CONCLUSION}

The goal of a diagnostic strategy is to perceive the appearance of a fault, to find the cause and then to deduce the action to follow in order to ensure the turbine's operational safety. Indeed, the use of methods based on the modeling of turbine variables makes it easier to identify their failures because the definition of the states is directly related to the turbine variables. For this purpose of fuzzy modeling of turbine failures, we opted for the interest in the use of Takagi-Sugeno models, which allows to consider another solution for monitoring vibrations using fuzzy techniques, emphasis on information extraction to characterize the states of this turbine. The findings are interesting and provide valuable performance for the diagnosis of the turbine examined.

\section{ACKNOWLEDGMENTS}

This work is supported by the Directorate General for Scientific Research and Technological Development (DGRSDT) and was carried out at the Applied Automation and Industrial Diagnostics Laboratory and at the Gas Turbine Joint Research Team in the University of Djelfa, Algeria

\section{REFERENCES}

1. Adel Alblawi. Fault diagnosis of an industrial gas turbine based on the thermodynamic model coupled with a multi feedforward artificial neural networks. Energy Reports. 2020;6:1083-1096. https://doi.org/10.1016/j.egyr.2020.04.029

2. Ahmed Zohair Djeddi, Ahmed Hafaifa, Abdellah Kouzou and Salam Abudura. Exploration of reliability algorithms using modified Weibull distribution: Application on gas turbine. International Journal of System Assurance Engineering and Management. 2017;8:1885-1894. https://doi.org/10.1007/s13198-016-0480-9

3. Chaoshun Li, Wen Zou, Nan Zhang, Xinjie Lai. An evolving $\mathrm{T}-\mathrm{S}$ fuzzy model identification approach based on a special membership function and its application on pump-turbine governing system. Engineering Applications of Artificial Intelligence 2018;69:93-103.

https://doi.org/10.1016/j.engappai.2017.12.005

4. Chuanlai Yuan, Yongyi Liao, Lingshuang Kong, Huiqin Xiao, Fault diagnosis method of distribution network based on time sequence hierarchical fuzzy petri nets. Electric Power Systems Research. 2021, 191:106870. https://doi.org/10.1016/i.epsr.2020.106870

5. Cristiano Hora Fontes, Hector Budman. A hybrid clustering approach for multivariate time series - A case study applied to failure analysis in a gas turbine. ISA Transactions. 2017; 71(Part 2): 513-529. https://doi.org/10.1016/j.isatra.2017.09.004

6. De-long Feng, Ming-qing Xiao, Ying-xi Liu, Haifang Song, Zhao Yang, Ze-wen Hu. Finite-sensor fault-diagnosis simulation study of gas turbine engine using information entropy and deep belief networks. Frontiers of Information Technology \& Electronic Engineering. 2016;17:1287-1304.

https://doi.org/10.1631/FITEE.1601365

7. Ehsan Mohammadi, Morteza Montazeri-Gh. A fuzzybased gas turbine fault detection and identification system for full and part-load performance deterioration. Aerospace Science and Technology. 2015;46:82-93. https://doi.org/10.1016/j.ast.2015.07.002

8. Ehsan Mohammadi, Morteza Montazeri-Gh. Active fault tolerant control with self-enrichment capability for gas turbine engines. Aerospace Science and Technology. 2016; 56: 70-89. https://doi.org/10.1016/j.ast.2016.07.003

9. Gareth L. Forbes, Robert B. Randall. Estimation of turbine blade natural frequencies from casing pressure and vibration measurements. Mechanical Systems and Signal Processing. 2013; 36(2): 549561. https://doi.org/10.1016/j.ymssp.2012.11.006

10. Habib Chaouki Ben Djoudi, Ahmed Hafaifa, Dalila Djoudi, Mouloud Guemana, Fault tolerant control of wind turbine via identified fuzzy models prototypes. Diagnostyka. 2020;21(3):3-13. https://doi.org/10.29354/diag/123220

11. Hang Gi Lee, Ju Hyun Shin, Chang-Ho Choi, Eunhwan Jeong, Sejin Kwon. Partial admission effect on the performance and vibration of a supersonic impulse turbine. Acta Astronautica. 2018; 145: 105115. https://doi.org/10.1016/j.actaastro.2018.01.025

12. Houman Hanachi, Jie Liu, Christopher Mechefske. Multi-mode diagnosis of a gas turbine engine using an adaptive neuro-fuzzy system. Chinese Journal of 
Aeronautics. 2018;31(1):1-9.

https://doi.org/10.1016/i.cja.2017.11.017

13. Imad Eddine Kaid, Ahmed Hafaifa, Mouloud Guemana, Nadji Hadroug, Abdellah Kouzou, Lakhdar Mazouz, Photovoltaic system failure diagnosis based on adaptive neuro fuzzy inference approach: South Algeria solar power plant. Journal of Cleaner Production. 2018;204:169-182. https://doi.org/10.1016/j.jclepro.2018.09.023

14. Jaroslav Václavík, Jan Chvojan. Torsion vibrations monitoring of turbine shafts. Procedia Structural Integrity. 2017;5:1349-1354. https://doi.org/10.1016/j.prostr.2017.07.197

15. Junkeon Ahn, Yeelyong Noh, Sung Ho Park, Byung Il Choi, Daejun Chang. Fuzzy-based failure mode and effect analysis (FMEA) of a hybrid molten carbonate fuel cell (MCFC) and gas turbine system for marine propulsion. Journal of Power Sources. 2017; 364: 226-233.

https://doi.org/10.1016/j.jpowsour.2017.08.028

16. Madhavan S, Rajeev Jain, Sujatha C, Sekhar AS. Vibration based damage detection of rotor blades in a gas turbine engine. Engineering Failure Analysis. 2014;46:26-39. https://doi.org/10.1016/j.engfailanal.2014.07.021

17. Marcin Adamowicz, Grzegorz Zywica. Advanced gas turbines health monitoring systems. Diagnostyka. 2018;19(2):77-87.

https://doi.org/10.29354/diag/89730

18. Mohamed Benrahmoune, Ahmed Hafaifa, Mouloud Guemana and XiaoQi Chen. Detection and modeling vibrational behavior of a gas turbine based on dynamic neural networks approach Journal of Mechanical Engineering. 2018; 68(3): 143-166. https://doi.org/10.2478/scjme-2018-0032

19. Mohammadreza Tahan, Elias Tsoutsanis, Masdi Muhammad, Abdul Karim Z.A. Performance-based health monitoring, diagnostics and prognostics for condition-based maintenance of gas turbines: A review. Applied Energy. 2017; 198: 122-144. https://doi.org/10.1016/j.apenergy.2017.04.048

20. Nadji Hadroug, Ahmed Hafaifa, Abdellah Kouzou, Ahmed Chaibet. Dynamic model linearization of two shafts gas turbine via their input/output data around the equilibrium points. Energy. 2017; 120: 488-497. https://doi.org/10.1016/j.energy.2016.11.099

21. Nadji Hadroug, Ahmed Hafaifa, Noureddine Batel, Kouzou Abdellah and Ahmed Chaibet. Active fault tolerant control based on a neuro fuzzy inference system applied to a two shafts gas turbine. Journal of Applied Artificial Intelligence. 2018; 32(6): 515-540. https://doi.org/10.1080/08839514.2018.1483114

22. Qingcai Yang, Shuying Li, Yunpeng Cao. A strong tracking filter based multiple model approach for gas turbine fault diagnosis. Journal of Mechanical Science and Technology. 2018; 32: 465-479. https://doi.org/10.1007/s12206-017-1248-0

23. Rodrigo Berrios, Felipe Núñez, Aldo Cipriano. Fault tolerant measurement system based on TakagiSugeno fuzzy models for a gas turbine in a combined cycle power plant. Fuzzy Sets and Systems. 2011; 174(11):114-130. https://doi.org/10.1016/j.fss.2011.02.011

24. Sierra-Espinosa F.Z., García J.C. Vibration failure in admission pipe of a steam turbine due to flow instability. Engineering Failure Analysis 2013; 27: $30-40$.

https://doi.org/10.1016/j.engfailanal.2012.08.011

25. Tianyang Wang, Qinkai Han, Fulei Chu, Zhipeng Feng. Vibration based condition monitoring and fault diagnosis of wind turbine planetary gearbox: A review. Mechanical Systems and Signal Processing. 2019;126:662-685.

https://doi.org/10.1016/j.ymssp.2019.02.051

26. Toufik Berredjem, Mohamed Benidir, Bearing faults diagnosis using fuzzy expert system relying on an Improved Range Overlaps and Similarity method. Expert Systems with Applications. 2018; 108: 134142. https://doi.org/10.1016/j.eswa.2018.04.025

27. Xin Wu, Yibing Liu. Leakage detection for hydraulic IGV system in gas turbine compressor with recursive ridge regression estimation. Journal of Mechanical Science and Technology. 2017; 31: 4551- 4556. https://doi.org/10.1007/s12206-017-0901-y

28. Yongjia Wu, Haifeng Zhang, Lei Zuo. Thermoelectric energy harvesting for the gas turbine sensing and monitoring system. Energy Conversion and Management 2018; 157:215-223. https://doi.org/10.1016/j.enconman.2017.12.009

29. Yu Zhang, Chris Bingham, Mike Garlick, Michael Gallimore. Applied fault detection and diagnosis for industrial gas turbine systems. International Journal of Automation and Computing. 2017; 14: 463-473. https://doi.org/10.1007/s11633-016-0967-5

30. Yuning Zhang, Xianghao Zheng, Jinwei Li, Xiaoze Du. Experimental study on the vibrational performance and its physical origins of a prototype reversible pump turbine in the pumped hydro energy storage power station. Renewable Energy. 2019; 130: 667-676.

https://doi.org/10.1016/j.renene.2018.06.057

Received 2020-08-04

Accepted 2020-10-30

Available online 2020-11-17

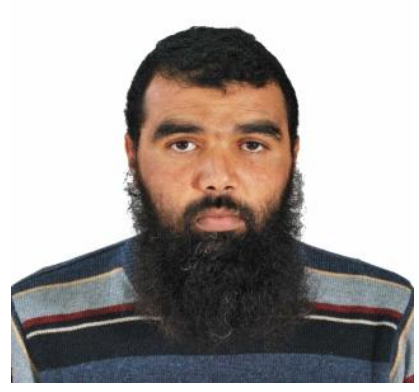

Mr ALILI Bachir was born on August 10, 1981 at Aflou LAGHOUAT. He obtained the state engineer degree on electromechanical engineering from Tiaret University and the Magister degree on high voltage from the University of Science and Technology of Oran on 2006 and 2011 respectively. $\mathrm{He}$ is currently preparing his $\mathrm{PhD}$ at Djelfa University. His researches are focusing on the fuzzy detection and fault localization caused by vibration in the gas turbine.

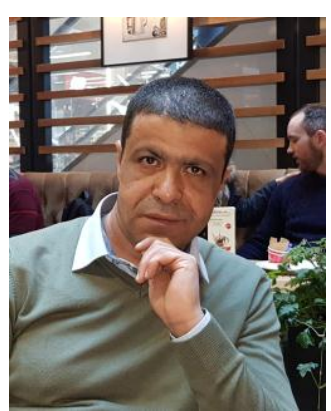

Professor

Ahmed HAFAIFA is a $\mathrm{PhD}$ and Full Professor in Industrial Process: Automation / Diagnosis and Reliability Engineering at the Science and Technology Faculty of the University of Djelfa, Algeria, where he is currently the Director of 
the Applied Automation and Industrial Diagnostic Laboratory of the University of Djelfa and the leader of the Gas Turbine Joint Research Team. He is the founder of these research entities, he is initiated and supported several international research projects in collaboration and innovation activities with the industrial sector.

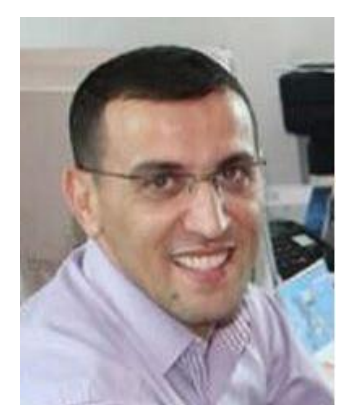

Dr Abdelhamid IRATNI is an associate professor in control engineering and automation systems at the electrical engineering department of the science and technology faculty of the University of Bordj Bou Arreridj (ALGERIA), where he was the founder of the department and has been a faculty member since 2004. He is the Chair of the scientific committee of the Electrical Engineering department since 2019 after serving as the head of the bachelor's and master's programs in automatic control for 3 years since 2015 and responsible for electromechanical engineering studies from 2012 to 2015. His current research areas include nonlinear filtering, estimation and control, diagnostic engineering, as well as automation and reliability of industrial systems. 